\title{
Beliefs and perception about mental health issues: a meta-synthesis
}

\author{
This article was published in the following Dove Press journal: \\ Neuropsychiatric Disease and Treatment \\ 31 October 2016 \\ Number of times this article has been viewed
}

\author{
Fahad Riaz Choudhry' \\ Vasudevan Mani ${ }^{2}$ \\ Long Chiau Ming ${ }^{3,4}$ \\ Tahir Mehmood Khan ${ }^{5}$ \\ 'Psychology Department, Jeffrey \\ Cheah School of Medicine and \\ Health Sciences, Monash University \\ Malaysia, Sunway City, Selangor, \\ Malaysia; ${ }^{2}$ College of Pharmacy, \\ Qassim University, Buraidah, \\ Al-Qassim, Kingdom of Saudi Arabia; \\ ${ }^{3}$ Vector-borne Diseases Research \\ Group (VERDI), Pharmaceutical \\ and Life Sciences CoRe, Universiti \\ Teknologi MARA, Shah Alam; ${ }^{4}$ Brain \\ Degeneration and Therapeutics \\ Group, Faculty of Pharmacy, \\ Universiti Teknologi MARA, Puncak \\ Alam, ${ }^{5}$ School of Pharmacy, Monash \\ University Malaysia, Sunway City, \\ Selangor, Malaysia
}

Background: Mental health literacy is the beliefs and knowledge about mental health issues and their remedies. Attitudes and beliefs of lay individuals about mental illness are shaped by personal knowledge about mental illness, knowing and interacting with someone living with mental illness, and cultural stereotypes. Mental health issues are increasing and are alarming in almost every part of the world, and hence compiling this review provides an opportunity to understand the different views regarding mental disorders and problems as well as to fill the gap in the published literature by focusing only on the belief system and perception of mental health problems among general population.

Method: The methodology involved a systematic review and the meta-synthesis method, which includes synthesizing published qualitative studies on mental health perception and beliefs.

Sample: Fifteen relevant published qualitative and mixed-method studies, regarding the concept of mental health, were identified for meta-synthesis.

Analysis: All the themes of the selected studies were further analyzed to give a broader picture of mental health problems and their perceived causes and management. Only qualitative studies, not older than 2010, focusing on beliefs about, attitudes toward, and perceptions of mental health problems, causes, and treatments were included in this review.

Results: The findings are divided into four major categories, namely, 1) symptoms of mental health issues, 2) description of mental health issues, 3) perceived causes, and 4) preferred treatment and help-seeking behavior. Each category contains themes and subthemes based on published studies.

Conclusion: The findings reveal multiple causes of, descriptions of, and treatment options for mental health problems, thereby providing insight into different help-seeking behaviors. Clarity is offered by highlighting cultural differences and similarities in mental health beliefs and perceptions about the causes of mental health problems. The implications of the studies and recommendations based on current findings are also discussed.

Keywords: mental health perception, mental health beliefs, mental health attitudes, meta-synthesis, mental disorders

\section{Introduction}

Mental health literacy refers to beliefs and knowledge of lay individuals about mental health problems/disorders and their treatment. It is identified by how lay individuals recognize and manage their specific disorders as well as estimate their outcomes and prognoses. ${ }^{1}$ Mental health is, to date, considered a neglected area in developing countries. Mental health problems can affect one out of every four people during their lives, by altering functioning, behavior, and thinking patterns. ${ }^{2}$

Attitudes and beliefs about mental illness are shaped by personal knowledge about mental illness, knowing and interacting with someone living with mental illness,
Correspondence: Tahir Mehmood Khan School of Pharmacy, Monash University Malaysia, Sunway City, Selangor, Malaysia Tel +60355144418

$\mathrm{Fax}+60355 \mathrm{I} 46364$

Email tahir.mehmood@monash.edu
Neuropsychiatric Disease and Treatment 2016:12 2807-2818

(c) (7) (ㄱ) 2016 Choudhry et al. This work is published and licensed by Dove Medical Press Limited. The full terms of this license are available at https://www.dovepress.com/terms.php cC) and incorporate the Creative Commons Attribution - Non Commercial (unported, v3.0) License (http://creativecommons.org/licenses/by-nc/3.0/). By accessing the work you hereby accept the Terms. Non-commercial uses of the work are permitted without any further permission from Dove Medical Press Limited, provided the work is properly attributed. For permission for commercial use of this work, please see paragraphs 4.2 and 5 of our Terms (https://www.dovepress.com/terms.php). 
cultural stereotypes about mental illness, media stories, and familiarity with institutional practices and past restrictions (eg, health insurance restrictions, employment restrictions, adoption restrictions). ${ }^{3,4}$

The cultural context is important when studying beliefs regarding mental health. The understanding of mental health and the interpretation vary from culture to culture. People's perception of illness explains their help-seeking behavior or lack thereof. ${ }^{5}$ It has been found that people stopped contacting with services and arranged for their own discharge, once they were diagnosed. ${ }^{6-8}$ Different regional studies revealed different views. Pacific Islanders, for example, considered mental illness as a result of family conflicts. ${ }^{9}$ According to a study on Jewish population, mental illness is seen as an opportunity to receive divine messages, a means of forgiveness, and to improve their souls. ${ }^{10}$ However, there are some cultures, mostly Southeast Asians, who perceive that supernatural forces/phenomena are responsible for mental health issues and consider them the result of wrath or denial of spirit or deities. ${ }^{11-13}$ This notion of supernatural or parapsychological phenomenon is not limited to Asia. Some Western cultures hold this idea too. A study conducted in Switzerland, with psychiatric patients, revealed that demons were considered the main cause of mental health problems. ${ }^{14}$ A South Asian study revealed that people there perceive mental illness as natural part of the suffering that is predestined for them.

Cultural differences exist regarding the etiology of mental health issues and the maintaining factors. Asian studies revealed the beliefs that somatic and organic factors lead to emotional problems and thus prefer physical treatment. ${ }^{15,16}$ Mental health problems and their causes are explained by Chinese culture as an imbalance of cosmic forces, and the preferred treatment is to restore the balance through interpersonal relationships, diet, exercise, and focusing on cognitions. ${ }^{16}$ Similar findings were revealed from another study in Nigeria. ${ }^{17}$

This review synthesized all the studies focusing on mental health beliefs, perceptions, and perceived etiological and treatment options. This review does not focus on any one regional study but encompasses all the studies showing common perceptions of mental health problems. It is significant to discuss and highlight the common themes of mental health perception in the different studies conducted in different cultures.

\section{Theoretical perspective}

Behavioral theorists conducted experiments to conclude that behaviors are acquired and learned through conditioning. ${ }^{18}$
However, cognitive theorists emphasize perception, thinking, belief systems, and other processes of remembering and learning for mental health. ${ }^{19}$ Likewise, sociocultural theory focuses on how acquisition of behaviors is influenced by cultural beliefs and attitudes, apart from peers and adult influences. ${ }^{20}$ According to Vygotsky's sociocultural theory, when a child is born, he/she has fundamental biological constraints on his/her mind; it is the culture that provides chances for learning, and as a result, children start using abilities of their mind in order to adapt their culture. ${ }^{21}$ Reasoned action and planned behavior theory proposes that individuals have the ability to act in rational ways and their behavior is influenced by their intentions. ${ }^{22}$ This theory asserts that most of the behaviors, including health behaviors, are under conscious control of an individual and behavior is best predicted by individuals' intentions to perform those behaviors. ${ }^{23}$ However, social cognitive theory puts forth that observations of an individual influence his/ her behavior through two kinds of modeling, direct modeling and symbolic modeling. ${ }^{22}$ According to Tudor's ${ }^{24}$ dual factor model, mental health and mental well-being have been presented as two separate concepts. This model views mental health as the presence or absence of mental illness or psychiatric symptoms, whereas mental well-being refers to emotionally prosperous and resilient at one end of continuum and weakening at the other end. According to this model, a person can have good mental well-being but deteriorating mental health. ${ }^{25}$ Rosenstock et al proposed the "Health Belief Model" that focuses on beliefs and attitudes to predict health behaviors. ${ }^{28}$ It understands the severity of illness and barriers to dealing with it in terms of how it is perceived by an individual. This model explains the causes of not utilizing health services by focusing on four constructs such as perceived susceptibility, perceived severity, perceived benefits, and perceived barriers. ${ }^{23}$ These four constructs have been seen to combine additively and affect the probability of executing certain health-related behavior. ${ }^{23}$ Perceived barriers have been shown to be the best predictor of behavior. ${ }^{26}$ Therefore, focusing on perception and belief systems is an important aspect of understanding any health-related issue. Specifically, discussing mental health, the term "mental health literacy" was introduced by Jorm et $\mathrm{al}^{27}$ and described as beliefs and knowledge regarding mental disorders, as well as awareness or information about their causes, risk factors, prevention, treatment, and help-seeking behavior. Focusing on the Health Belief Model of Rosenstock et al, ${ }^{28}$ this review has targeted the cognitive elements of perception and belief systems. 


\section{Aim of the study}

The above-mentioned findings and theories provide the grounds to consider the significance of studying belief systems and perception regarding mental health problems. This study was planned in view of the scarcity of published reviews on belief systems regarding mental health problems. Mental health issues are increasing and are alarming in almost every part of the world, ${ }^{29-32}$ as well as a lack of knowledge regarding prevention and progress of mental illnesses, relevant information about seeking help, and treatment options has been reported. ${ }^{27}$ It has been seen that only a limited number of individuals seek professional help for mental disorder, ${ }^{33-35}$ and hence the understanding of mental health literacy automatically gains importance. So this review provides an opportunity to understand different views regarding mental disorders and problems, which will be equally beneficial for researchers, professionals, and the general public to access fresh studies on the subject matter.

Furthermore, the aim of this review is to present a holistic picture about variations in mental health perceptions and beliefs among different masses and cultures. This review is not targeted at any specific culture or profession but encomand discusses generally the perception and beliefs regarding mental health problems that people commonly encounter. We also aim to find out the potential belief systems regardmental health problems and their causes and treatments, which are discussed and published in different studies using different qualitative methods.

\section{Methodology Meta-synthesis}

Meta-synthesis is a relatively new qualitative technique, used for amalgamation of qualitative studies for the purpose of exploring or developing a theoretical model that can explain findings of different studies on a similar topic. ${ }^{36}$ In this study, a systematic review and meta-synthesis method was used for gathering qualitative studies on mental health perception and beliefs.

\section{Systematic literature review}

The literature consisted of qualitative studies on mental health perception and beliefs searched for using keywords. The key search terms and their truncated variance were mental health beliefs, mental health perception, attitudes toward mental health issues, experiencing mental health problems, and mental health literacy.

\section{Databases}

The key terms were used to find the desired articles in a number of databases (Table 1) including PsycINFO,
GoogleScholar, Scopus, Cochrane database, and PubMed. After generation of key terms, some known articles and citations from those articles were identified. Search terms focusing on the description, symptoms, causes, and diagnosis of the common mental disorders of depression and anxiety were also used to find appropriate articles. These identified articles were further filtered using the terms "mental health beliefs", "mental health perception", "qualitative studies", "qualitative methodology", "thematic/content analysis", and "interpretative phenomenological analysis". Similarly, these searches were done through PsycINFO, GoogleScholar, Scopus, Cochrane database, and PubMed.

\section{Inclusion criteria}

The studies included were qualitative studies from 2010 or later focusing on at least one or all of the following concepts: mental health beliefs/attitudes/perception, causes and etiological factors, description of mental health issues, and perceived symptoms and treatment. Mixed-method studies were included whose qualitative part focused on these concepts.

\section{Exclusion criteria}

All quantitative studies were excluded, as our aim was to conduct a meta-synthesis approach, that is, synthesis of qualitative studies. All the meta-analysis and meta-synthesis studies were also excluded. Studies using ethnography and grounded theory methodology were excluded as the current synthesis focused on those studies in which beliefs and perceptions of people regarding mental health issues were explored.

\section{Procedure and synthesis of themes}

The key terms were identified, and a systematic search of articles was done using relevant databases (Table 1). Data were extracted using this systematic search of articles. Identified articles were selected and were further screened out for inclusion or exclusion depending upon our described criteria (Figure 1). Data in the form of themes, extracted

Table I Search terms and databases used to retrieve the articles

\begin{tabular}{lll}
\hline Search terms and results & Databases & Result \\
\hline Mental health (I30) (total) $=$ & PsychINFO, & I5 articles were \\
mental health perception (8) & GoogleScholar, & found relevant \\
mental health beliefs (13) & Scopus, & according to inclusion \\
mental health attitudes (13) & PubMed, & criteria \\
mental health treatment (19) & Cochrane & \\
mental health causes (I7) & \\
mental health problems (3I) & \\
mental health issues (2I) & \\
mental health literacy (8) & \\
\hline
\end{tabular}




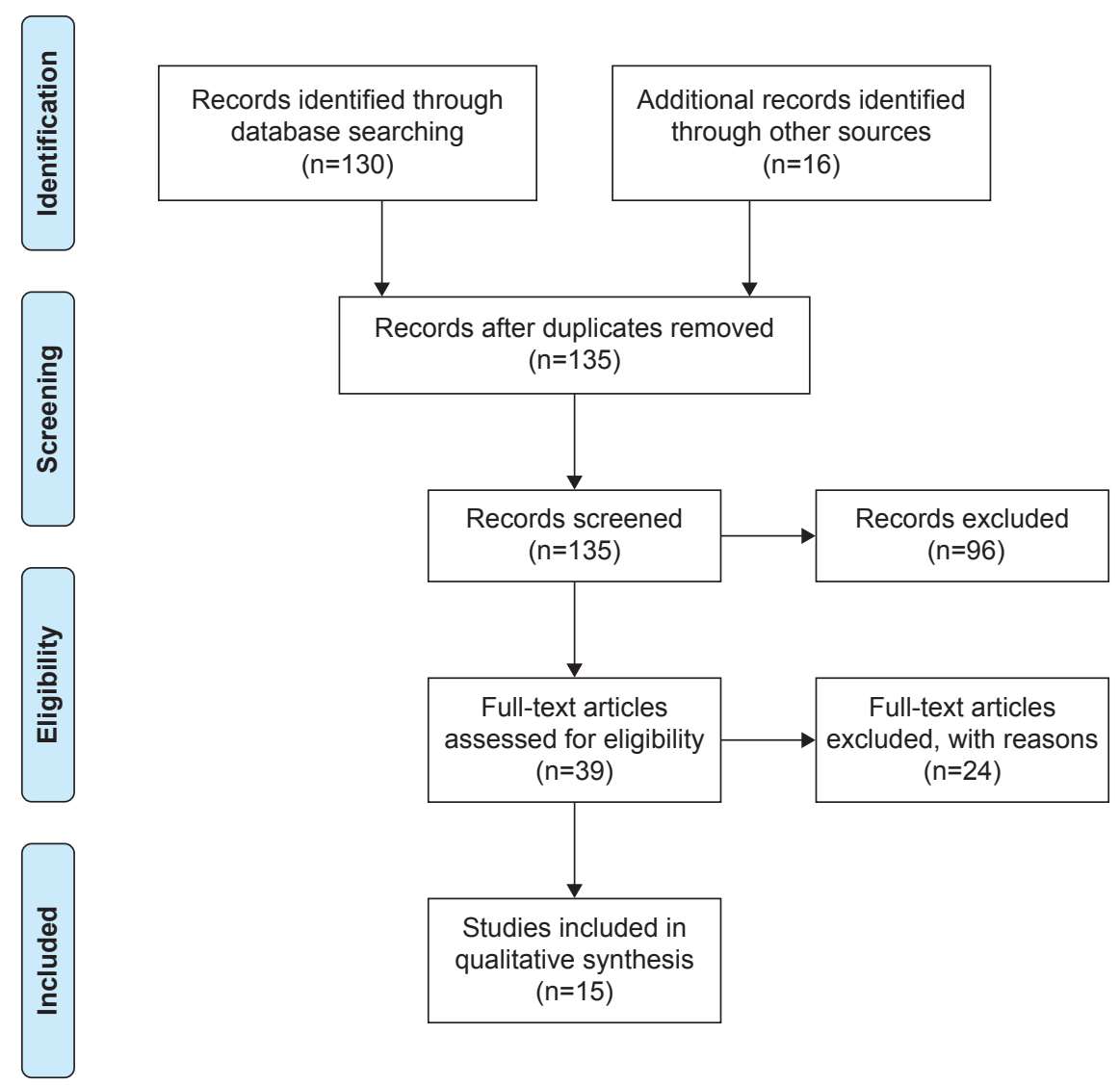

Figure I PRISMA flow diagram.

from different studies, were synthesized and are presented in Table 2. The analysis process included synthesis of themes and analyzing them further to achieve the objectives of the study, namely, focusing on studies exploring mental health beliefs, ideas, perception, etiological factors, and treatment preferences. So themes extracted from the studies focusing on these areas were further transformed into categories showing similar themes and concepts. Every category of themes has some subcategories or a number of similar themes derived from different studies. Second, themes were interpreted using the author's perspectives and also by considering and stating the interpretations in different studies from the perspectives of their authors. Newly formed categories of themes were analyzed using verbatim translation, comparing participants' description, keywords, and terms reflecting similarities and differences.

\section{Quality appraisal}

Quality appraisal ensures the necessary reliability and accuracy between interpretations done by authors and the primary data. Previous meta-synthesis studies ${ }^{37-39}$ used quality appraisal tools, and we followed those studies in using this technique. The quality appraisal consisted of the following dimensions: 1) adequacy and suitability of research design to the main research question and 2) appropriateness to present primary data in relation to analysis. It also included systematic data collection procedure and well-documented data collection.

\section{Ethics}

This was a review study with no humans directly involved. However, the authors met some ethical requirements by respecting the moral rights of authors, whose studies were included by clearly presenting their findings, thereby avoiding intentional manipulation or falsification of data.

\section{Results}

Results are presented in four broad categories: 1) symptoms of mental health issues, 2) description of mental health issues, 3) perceived causes, and 4) preferred treatment and help-seeking behavior. Each category contains themes and subthemes. The categories are based on the repeated themes in different studies.

\section{Symptoms}

Different qualitative studies suggested different symptoms. However, most of the studies focusing on symptoms and their idiosyncratic understanding can be divided into the following two themes: 1) mood related and 2) behavioral symptoms. 


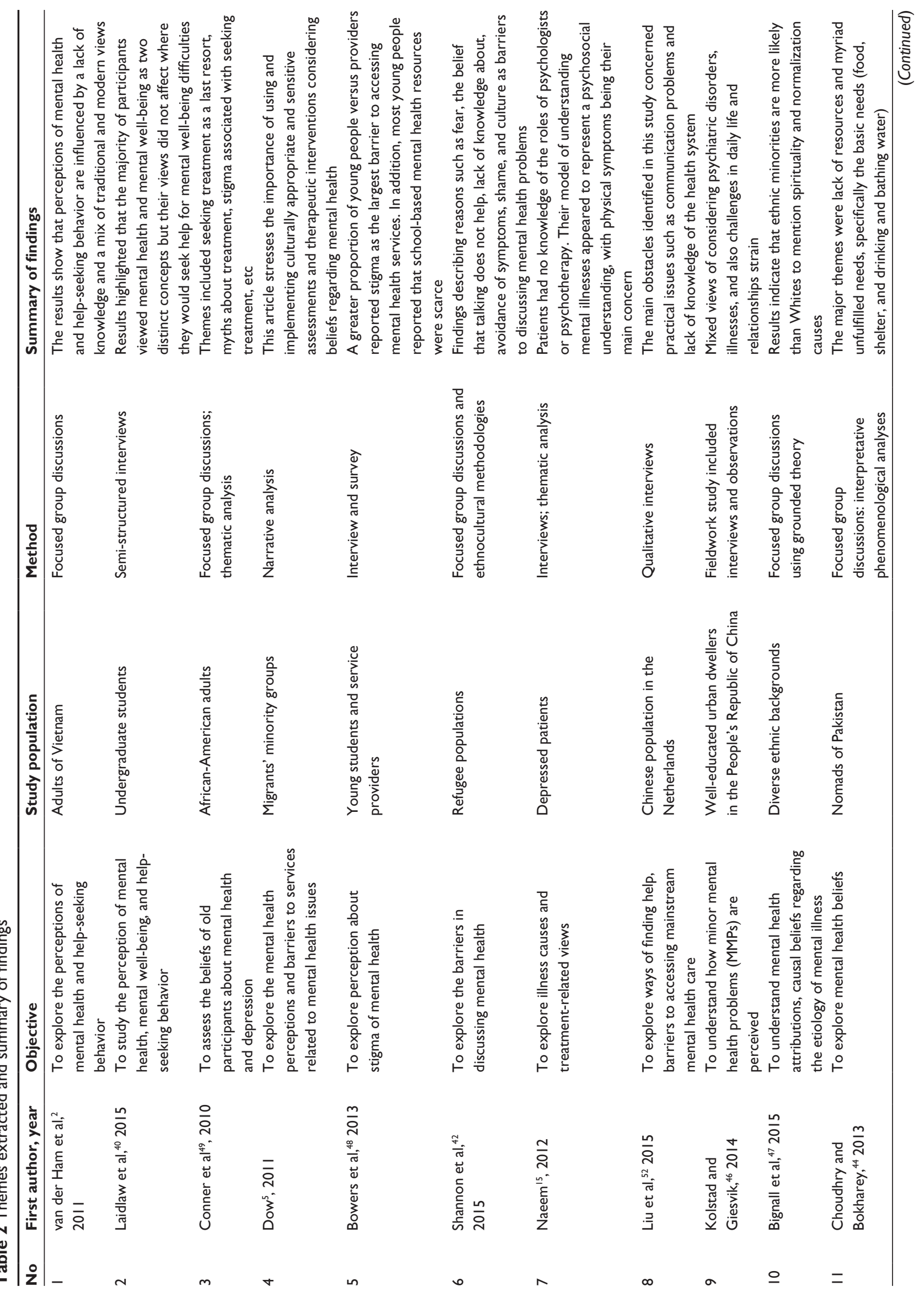




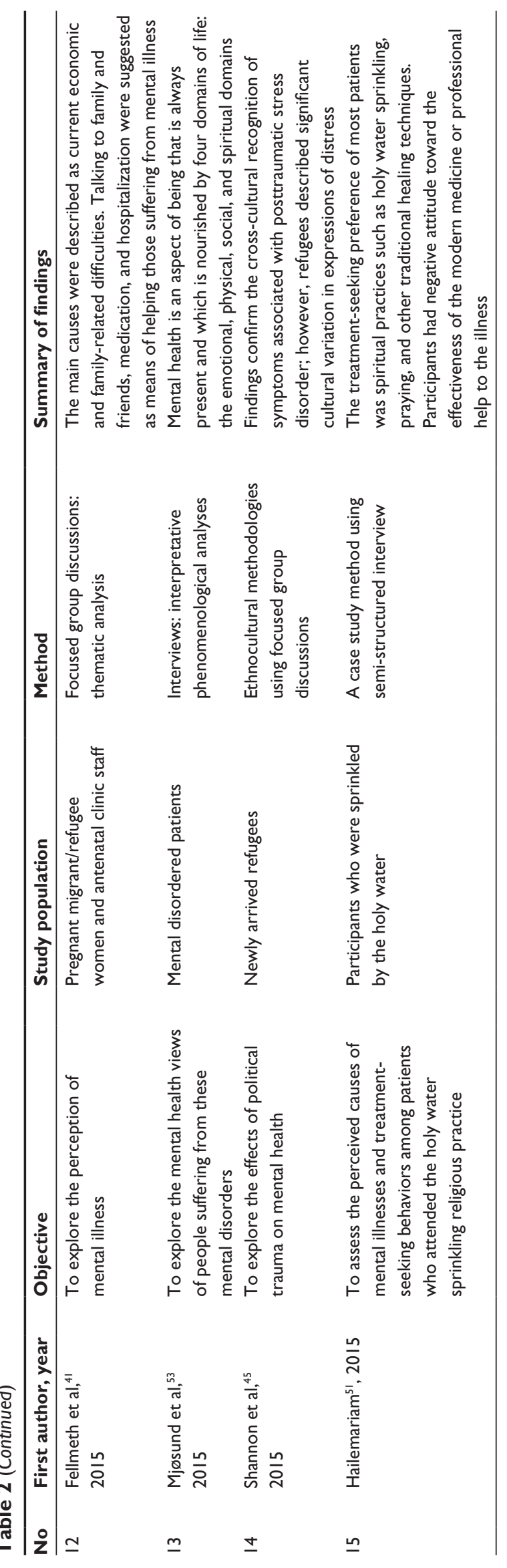

\section{Mood related}

Emotions and mood shifts were considered as significant indicators of mental health problems. Findings from different studies suggested disturbing emotions as a manifestation of mental health problems. Participants in a study by Laidlaw et $\mathrm{al}^{40}$ discussed the symptoms of mood shifts and the role of stress as important symptoms, and sadness, low mood, anger, and lack of attention and concentration were also attributed to mental health problems. These findings are consistent with previous studies and with diagnostic criteria for mood and anxiety disorders given by Diagnostic and Statistical Manual for Mental Disorders, fifth edition. These symptoms are also present with other symptoms in diagnostic criteria of psychotic disorders.

\section{Behavioral symptoms}

Previous studies ${ }^{2,41,42}$ highlighted the behavioral symptoms of mental health issues. A pattern of consensus can be found in all these studies regarding the existence of deviant behavior specifically revealing irrelevant talk, inappropriate behavior, and self-talk, self-laughter, and crying as some major behaviors identified as abnormal. Studies also revealed physical symptoms of body aches and headaches and escaping behavior, that is, avoiding or escaping from difficult situations, in addition to the previously discussed symptoms. These findings are consistent with the behavioral theories of psychology that reject the traditional belief in unconscious behavior and replace it with the notion that problematic behaviors could be learned behaviors. ${ }^{43}$

\section{Description}

The second main category is the description of mental health problems. This includes the themes that were extracted from the excerpts of participants from different studies and discussed as a description of mental health problems.

\section{Emotional, behavioral, and physical}

While discussing the emotional aspects of mental issues and well-being, happiness was described as a concept related to "healthy" and "well-being", whereas "mental health" referred to serious psychiatric illnesses such as schizophrenia and depression. ${ }^{44}$ Dow $^{5}$ identified shame and stigma as the reactions to social pressures whereas demands as a description and attribution of mental health issues. Furthermore, studies ${ }^{15,44,45}$ revealed the emotional, behavioral, and physical aspects of mental health issues and described mental health problems in all three areas. In the emotional domain, fear, guilt, anger, helplessness, pain, anxiety, and sadness were 
the major discussed features of mental anomalies. Behavioral descriptions ranged from inappropriate behavior, isolation, and wandering to self-talk and poor hygiene/poor self-care. Physical descriptions of body aches, headaches, stomach aches, and sleeplessness were discussed. Some very different terms were introduced for mental health problems in one study focusing on the perception and understanding of mental health issues, for example, "heart problems" did not refer to cardiac problems but were associated with heartbreaks through relationship conflicts, and these problems were considered as obstacles to well-being and optimal functioning. ${ }^{46}$

\section{Normalization, trauma, and stress}

Another theme extracted from different studies referring to the description of mental health issues is normalizing illness. Kolstad and Gjesvik ${ }^{46}$ found that participants believed that depression is a minor mental health problem and used this normalization strategy to describe the mental illness. Normalizing and underestimating symptoms of mental issues were also revealed by Bignall et $\mathrm{al}^{47}$ and further discussed and described mental health issues in terms of trauma and stress. Similarly, other studies ${ }^{2,40,44}$ described mental health issues in the context of stress and poor coping.

\section{Perceived causes of mental health problems}

Almost all the selected studies discussed and focused on beliefs of participants about the perceived causes of their mental health problems.

\section{Psychosocial and environmental factors (stress)}

Most of the studies, when discussing causes of mental health issues, described psychological factors such as unhappiness, low self-esteem, rejection, overthinking, self-downing and blaming, anxiety and worry, and conflicts in familial and other interpersonal relationships..$^{2,15,41,44,48}$ Socioeconomic factors of poverty, lack of earning opportunities, unfulfilled basic and secondary needs, ${ }^{44}$ racial and ethnic discrimination, ${ }^{49}$ and economic injustice were also believed to be important factors resulting in mental health problems. ${ }^{45}$ Kolstad and Gjesvik ${ }^{46}$ explored the beliefs regarding causes of mental health and mostly revealed social factors including societal changes, adopting Western values, decrease in traditional values, and indigenous approaches of living a simple life. Similarly, Dow ${ }^{5}$ identified lacking direction and purpose in life as an important factor leading to mental health problems. In a similar fashion, Fellmeth et $\mathrm{al}^{41}$ attributed mental health problems to a lack of social support, familial conflicts, and economic problems. Challenging events, trauma, and everyday life stresses were also identified as some important factors for the development of mental health problems. ${ }^{2,15,40,41}$ Stress is also believed to be a significant cause of mental health problems discussed in these studies. These findings can be related to Nguyen ${ }^{50}$, where similar perceptions about the causes of mental illnesses were discussed, and reflect the belief system of Vietnamese people. Nguyen discussed the role of stress in terms of studying or thinking too much, which results in mental health issues. These findings reflect culturally specific beliefs about mental health in connection with stress, excessive mental exertion, and overload.

\section{Spiritual and supernatural causes}

The second most frequent cause of mental health problems cited in the selected studies was spiritual. Mental disorders were interpreted as a result of the wrath of God, curses, and evil spirits. ${ }^{51}$ People attributing them to spirits was found in a number of studies. ${ }^{41,44,47,51,52}$ Within a spiritual and supernatural domain, there was some distinction as these aforementioned studies attributed mental health issues to negative forces or the result of the anger of God, while other studies revealed the beliefs of people attributing mental health problems to some blessings and spiritual connection with God and getting special attention from nature. ${ }^{44,53}$

\section{Biomedical and genetic causes}

Some studies revealed people's belief in biomedical and genetic causes of mental disorders. Some participants discussed that genes played an important role, and mental disorders were attributed to genetic reasons. ${ }^{51}$ According to Dow, ${ }^{5}$ participants discussed and believed that there were biological reasons for the development of mental health problems. Similarly, in addition to largely discussing spiritual causes, some participants in another study noted medical reasons. ${ }^{52}$ Even some marginalized populations had awareness regarding medical causes despite their belief in the spiritual causes of mental illnesses. ${ }^{44}$

\section{Treatment and help-seeking behavior}

Different studies revealed different etiological factors for mental disorders and mental health problems. Similarly, they also recommended treatment options for managing and overcoming mental health problems. Most of the studies that identified more than one cause also recommended more than one treatment model. 


\section{Psychological and psychiatric}

Treatment with psychotherapeutic and psychiatric medicines, and hospitalization following medical treatment, was discussed in many studies. ${ }^{2,47,52}$ However, there were some findings pointing only to psychiatric and medicinal treatment and not psychological treatment. ${ }^{2,15,44}$ On contrary, there were findings where participants discouraged professional psychological or psychiatric treatment and advised people not to seek any treatment unless the severity was extremely high. Even then, participants preferred to employ the traditional treatment of willpower strategies. This treatment resistance was due to culturally sanctioned ideas in an African background, where mental health treatment is stigmatized and there were many myths regarding mental health problems. Similar findings were presented in previous studies exploring perceived causes and treatment of mental health issues among Africans and AfricanAmericans, which revealed that they were not likely to access mental health services and preferred to use informal sources of care ${ }^{54-56}$ However, there were some findings to the contrary among older African-Americans, who showed willingness to seek professional psychotherapeutic and psychopharmacological treatment. ${ }^{46}$ Other studies validated professional mental help, preferring psychological treatment over medicinal treatment. ${ }^{49}$

\section{Spiritual treatment}

Recommending spiritual treatment was another significant and widely occurring belief in many studies. Consulting spiritual healers, ${ }^{44}$ practicing prayers, recitation of sacred texts, and use of holy water were found to be the treatment options. ${ }^{41,51,53}$ These practices reflected beliefs in supernatural forces and also the use of religious sacred texts and verses to overcome problems. Mostly, such beliefs are found in marginalized populations. ${ }^{44}$ However, participants' belief in religious practices and spiritual treatment cannot be generalized to one socioeconomic class, as some of the abovementioned studies where the subject population was not in a low economic position reflect the same belief system. There is some similarity between the kind of spiritual treatment incurred and discussed by different studies, as the beliefs of evil possession and spirits are common to different faiths and religions and so are their treatment options.

One treatment procedure discussed in one of the selected studies $^{51}$ of this review is included here, because similar procedures are discussed in other studies, where the name or wordings for the evil spirit may vary but the concept is more or less the same. If disease is believed to be caused by spirits, evil possessions, genies, or similar phenomenon, the common method for treatment is often the use of smoke within a room. This act is considered to undo the attack of the evil spirits on the affected person. Furthermore, the patient is recommended to be near the smoke, and the priest or healer repeatedly asks the patient to converse with the evil inside and give it commands to leave the body. ${ }^{51}$ Consulting faith or spiritual healers was discussed in other studies as well. ${ }^{41,44,53}$ These findings are not restricted to one faith or religion but occur across different cultures, faiths, religions, and ethnicities. These studies focused mostly on Christian cultures and African cultures. But there are findings from Muslim Arab cultures with similar practices of consulting faith healers and believing in the spiritual causes of mental health problems..$^{57,58}$

\section{Social support and significant others}

Consulting and sharing the problems with significant others, that is, family, friends, and loved ones, was another theme extracted from many studies. . $, 5,40,41,52$ Some studies strongly suggested social support as a treatment option unless the mental health problem was very severe. ${ }^{46}$ Empathy shown by family, friends, and neighbors serves as resilience or protective factors against the mental health issues, and such emotional support from social relationships is believed to be a significant factor in treating mental health issues. ${ }^{5,41}$ Social support can be explained with different angles and viewpoints. In some cases, the support refers to the function or purpose of the interaction, for example, enjoying performing desirable tasks, sharing love, or exchanging information. It also refers to the structure of certain relationships or even to the origin of the support, for example, family, partner, or friends.

\section{Barriers to treatment}

There were some studies that also discussed barriers to seeking help for mental health problems and identified some barriers including cultural barriers such as stigma and taboo. Stigma of madness was the most significant barrier to seeking mental health help. ${ }^{5,42,48}$ Lack of knowledge and awareness about mental health treatment was the second most occurring barrier. ${ }^{15,42,44,47,48,59}$ Some economic and legal and linguistic barriers were also identified. ${ }^{44,52}$ Ethnicity was found to be an important barrier in accessing mental health services. Some marginalized or stigmatized communities in a specific culture that were discriminated against discussed prejudice as a significant barrier in seeking help for mental health problems. ${ }^{46}$ 
Some participants did not state clearly about barriers in seeking mental health support but indirectly discussed their deprived and unfulfilled basic and secondary needs, poverty, and rejection from society as marginalized groups, which surely serve as big obstacles to consulting professional mental health-related services. ${ }^{44,52}$ Lack of awareness about mental health problems and its treatment was another important barrier in consulting professional mental health help. A significant number of participants in many studies reported a lack of awareness about treatment options and availability. ${ }^{15,44,48}$

\section{Discussion}

The aim of this study was to ascertain the beliefs and perception about mental health issues prevailing among different populations and cultures, published in different qualitative studies. Synthesizing all those findings of selected articles, the results are presented in the four main categories: 1) symptoms of mental health issues, 2) description of mental health issues, 3) perceived causes, and 4) preferred treatment and help-seeking behavior. These categories are formed considering the repetition of similar themes discussed across different studies. Symptoms of mental health issues are perceived in terms of mood and behavior and discussed largely by the participants. Viewing mental health issues in terms of symptomatology is in harmony with the dual factor model as participants of various studies discussed distinct behavioral and mood symptoms for mental illness. ${ }^{60}$ Mood shifts and disturbing emotional conflicts were discussed as important symptoms of mental health issues. Studies revealed implying behavioral and mood-related symptoms such as irrelevant talk, inappropriate behavior, self-talk, crying and sadness, low mood, anger, and lack of attention and concentration symptoms..$^{15,40,49}$ This can be related with symptoms of mood disorders and common mental disorders of depression, anxiety, and some of the negative symptoms of psychotic disorders. ${ }^{61}$ Therefore, the emerged theme is in harmony with the dual factor model aspects as various studies have brought forth distinct behavioral and mood symptoms as a result of mental illnesses.

Normalization, stress, and trauma are described by participants in different studies and considered as another theme in this review. Psychosocial stress is seen as among the most severe health problems in the 21 st century. ${ }^{62}$ Previous studies assessed the positive relationship between stress and mental illnesses. ${ }^{63-65}$ An increase in the number of mental illnesses owing to psychosocial stressors has been reported. ${ }^{66,67}$ Researchers have put forth psychosocial models to elaborate how psychological mechanisms and stress influence mental health. ${ }^{68-70}$ It has also been highlighted that personal resources of an individual either avert or exacerbate mental illness through two mechanisms, either through direct activation of coping mechanisms of an individual or through interactively shielding against stress. ${ }^{71,72}$ This is a common belief found among different people from different walks of life and is also implied by the psychosocial model of disease that views stress and trauma-related stress as significant factors in the development of mental health problems. ${ }^{73}$ Therefore, the present findings are supported by existing literature on psychosocial models of stress and distress, which propose that psychological mechanism plays a vital role in mental health of an individual.

This study also described the treatment options and causes that participants in different studies discussed in terms of mental health problems. Generally, it is worth mentioning here that the pattern found in the causes and treatment is consistent with the approach participants adopted. It means that some participants believed in scientific causes and treatments, while it varies from person to person and among different groups and cultures. It must also be mentioned here that the treatment approach and causes of mental illness adopted by participants were in harmony with the group they belonged to or the culture they lived in. Some participants discussed psychological and psychiatric treatment as their preferred choice for mental health problems so we can ascertain that they are following a scientific approach to mental health problems. However, there are studies ${ }^{53,56,61,65,73}$ discussing supernatural causes as well as consulting faith healers, religious scholars, and/or spiritual healers for treatment of mental health issues, as participants believed in the supernatural and spiritual etiology for mental disorders and problems. The beliefs related with supernatural causes of mental illnesses and opting related modes of treatment were prevalent across respective cultures. Such perceptions of participants are consistent with Vygotsky's sociocultural theory, ${ }^{20}$ which proposes that culture not only shapes the behavior of individuals but also modifies their behavior to adapt in a certain culture. These findings are well related with the spiritual model of illness causation, ${ }^{74}$ which refers to the spiritual etiology of mental health problems and the same treatment discussed under the spiritual treatment theme of our study.

Another important theme among the findings of the published studies is the role of social support and significant others, that is, family members and friends. Participants from different studies discussed the importance of social support for overcoming the mental health problems. ${ }^{2,5,40,41,52}$ 
This is in harmony with relationship perspective of social support that proposes how social support influences health and how relationship processes influence an individual, it is interrelated; effects of social support on health as well as relationship processes co-occur. ${ }^{75}$

The current synthesis study is helpful for clinicians devising management plans for patients with mental health problems as it focuses on the beliefs that are most commonly present in different cultures, thus giving insight to psychotherapists to better plan their interventional plans for patients. Also the recommendations based on the findings could be forwarded to policymakers to devise some policies for awareness-raising campaigns among the general population, as well as in health and educational settings.

Overall, different qualitative studies focused and discussed varying causes, description, and treatment options reflecting different help-seeking behaviors. Based on the findings of this study, we can conclude that mental health beliefs and perceptions vary from culture to culture, but there do exist some similar beliefs and perceptions about the descriptions and causes of mental health problems. Some studies focused on mental health perceptions and variation within the concept of mental health and mental well-being as two distinct concepts and also discussed stigma associated with seeking help for mental health. ${ }^{40}$ As in this study, the population was medical students and other students, so we can ascertain that they were a conscious population and cognizant of mental health-related scientific information. Similarly, where the population is general adults such as the one in Vietnam, the findings described symptoms corresponding to those mentioned in the Diagnostic and Statistical Manual for Mental Disorders, and most of the participants not only discussed the priority of medical treatment but also emphasized traditional concepts by few participants. So overall, there were mixed views ranging from medical treatment through to the family's role and traditional values and also some ignorance regarding adequate information. ${ }^{2}$ Again we can see these mixed views as varying perceptions among the lay population about mental health beliefs, and these views are related with behavioral descriptions discussed in psychological theories.

There are differences observed if we look at studies focusing on African-Americans, Bhutanese, Burmese, Ethiopians, Somalians, and Pakistanis. The cultural differences highlighted in a few studies concluded that participants seek mental health help as a last resort and found a stigma associated with treatment and some myth-related beliefs of mental health perception and treatment. Studies such as this provide the grounds to work on awarenessraising regarding mental health perceptions where myths are believed rather than scientific explanations. Likewise, in some other studies, ${ }^{41,42,44,47,51}$ despite the discussions of psychosocial and medical causes, spiritual phenomenon and supernatural causes are believed to be the major causes of mental health problems, and similar remedies were recommended.

Considering differing concepts ranging from disease and medical model to social issues and spiritual beliefs, there are mixed kinds of beliefs in almost every study. This study has some implications for mental health practitioners as it gives a broader picture of mental health perceived causes. The practitioners can devise management plans considering these varying beliefs, and to enhance awareness, some massive campaigns could be initiated especially for marginalized and ignored populations as well as for general public to develop insight regarding scientific, medical, and psychosocial causes and treatment.

\section{Conclusion}

Apart from normalization, stress, and trauma, the importance of social support was brought to light. Analysis of previous qualitative literature revealed a variety of cultural similarities and differences in causes and description of mental disorders, ranging from spiritual to medical causes and from social to psychological causes. Likewise, preferred modes of treatments also varied from person to person and from one group to another, and they comprised both scientific and unscientific methods. Owing to the importance given to various beliefs held across cultures, a study such as this can prove valuable to psychotherapists in designing management plans for patients. The findings of the present study can assist policymakers in launching campaigns that can increase awareness of biopsychosocial causes and treatments of mental disorders, among the general population as well as in health and educational settings.

\section{Limitations}

The main limitation was exclusion of quantitative studies on mental health beliefs and those studies that are not published or are ongoing. An important limitation is the exclusion of articles fulfilling the criteria of the study but not available in English (no translation service was available). Selected studies were not restricted to any specific culture, sex, religion, ethnicity, or geographical region and therefore discussed and synthesized the beliefs of people from different communities at a broader/general level. 


\section{Acknowledgments}

This work was supported by Academic and Research Assimilation grants: 600-RMI/DANA 5/3/ARAS (64/2015). The authors would like to express their gratitude to Ministry of Higher Education and Universiti Teknologi MARA (UiTM), Malaysia, for their financial support. The funders had no role in study design, data collection and analysis, decision to publish, or preparation of the manuscript.

\section{Disclosure}

The authors report no conflicts of interest in this work.

\section{References}

1. Suhail K. A study investigating mental health literacy in Pakistan. $J$ Ment Health. 2005;14(2):167-181.

2. van der Ham L, Wright P, Van TV, Doan VD, Broerse JE. Perceptions of mental health and help-seeking behavior in an urban community in Vietnam: an explorative study. Community Ment Health J. 2011;47(5): 574-582.

3. Corrigan PW, Markowitz FE, Watson AC. Structural levels of mental illness stigma and discrimination. Schizophr Bull. 2004;30(3):481-491.

4. Wahl O. Depictions of mental illnesses in children's media. $J$ Ment Health. 2003;12(3):249-258.

5. Dow HD. Migrants' mental health perceptions and barriers to receiving mental health services. Home Health Care Manag Pract. 2011; 23(3):176-185.

6. Tehrani E, Krussel J, Borg L, Munk-Jorgensen P. Dropping out of psychiatric treatment: a prospective study of a first-admission cohort. Acta Psychiatr Scand. 1996;94:266-271.

7. Edlund MJ, Wang PS, Berglund PA, Katz SJ, Lin E, Kessler RC. Dropping out of mental health treatment: patterns and predictors among epidemiological survey respondents in the United States and Ontario. Arch Gen Psychiatry. 2002;159:845-851.

8. Young AS, Grusky O, Jordan D, Belin TR. Routine outcome monitoring in a public mental health system: the impact of patients who leave care. Psychiatr Serv. 2000;51:85-91.

9. Douglas KC, Fujimoto D. Asian Pacific elders: implications for health care providers. Clin Geriatr Med. 1995;11:69-82.

10. Selekman J. People of Jewish heritage. In: Purnell LD, editor Transcultural Health Care: A Culturally Competent Approach. 4th ed. Philadelphia, PA: FA Davis; 2012:338-356.

11. Khan T, Hassali M, Tahir H, Khan A. A pilot study evaluating the stigma and public perception about the causes of depression and schizophrenia. Iran J Public Health. 2011;40(1):50-56.

12. Kinzie JD. Overview of clinical issues in the treatment of Southeast Asian refugees. In: Owan TC, editor. Southeast Asian Mental Health: Treatment, Prevention, Services, Training, and Research. Washington, DC: National Institute Mental Health; 1985:113-135.

13. Mishra SI, Lucksted A, Gioia D, Barnet B, Baquet CR. Needs and preferences for receiving mental health information in an African American focus group sample. Community Ment Health J. 2009;45(2): 117-126.

14. Pfeifer S. Belief in demons and exorcism in psychiatric patients in Switzerland. Br J Med Psychol. 1994;67(pt 3):247-258.

15. Naeem F, Ayub M, Kingdon D, Gobbi M. Views of depressed patients in Pakistan concerning their illness, its causes, and treatments. Qual Health Res. 2012;22(8):1083-1093.

16. Sue S. Mental health: Conforming critical health issues of Asian and Pacific Islander Americans. Thousand Oaks, CA: Sage; 1994.

17. Adebowale TO, Ogunlesi AO. Beliefs and knowledge about aetiology of mental illness among Nigerian psychiatric patients and their relatives. Afr J Med Med Sci. 1999;28(1-2):35-41.
18. Jones-Smith E. Theories of Counseling and Psychotherapy: An Integrative Approach. USA: SAGE Publications, Inc; 2012.

19. Cherry K [webpage on the Internet]. Psychological Theories; 2014. Available from: http://psychology.about.com/od/psychology101/u/ psychology-theories.htm. Accessed August 11, 2016.

20. Vygotsky L. Thought and Language. Cambridge, MA: The MIT Press; 1986.

21. Shaffer DR. Social and Personality Development. Belmont, CA: Wadsworth; 2009.

22. Lakhan SE [webpage on the Internet]. Theories on Health Behaviors; 2006. Available from: http://brainblogger.com/2006/03/19/bpstheories-on-health-behaviors/. Accessed August 11, 2016.

23. Sutton $\mathrm{S}$ [webpage on the Internet]. Health Behavior: Psychosocial Theories; 2002. Available from: http://userpage.fu-berlin.de/ schuez/ folien/Sutton.pdf. Accessed August 11, 2016.

24. Tudor K. Mental Health Promotion: Paradigms and Practice. London: Routledge; 1995.

25. Wang X, Zhang D, Wang J. Dual-factor model of mental health: surpass the traditional mental health model. Psychology. 2011;2(8):767-772.

26. Janz NK, Becker MH. The health belief model: a decade later. Health Educ Q. 1984;11:1-47.

27. Jorm AF, Korten AE, Jacomb PA, Christensen H, Rodgers B, Pollit P Mental health literacy: a survey of the public's ability to recognize mental disorders and their beliefs about the effectiveness of treatment Med J Aust. 1997;166:182-186.

28. Rosenstock IM, Strecher VJ, Becker MH. Social learning theory and the health belief model. Health Educ Q. 1988;15(2):175-183.

29. Klerman GL, Weissman MM. Increasing rates of depression. JAMA. 1989;261(15):2229-2235.

30. Kessler RC, Berglund P, Demler O, Jin R, Merikangas KR, Walters EE. Lifetime prevalence and age-of-onset distributions of DSM-IV disorders in the national comorbidity survey replication. Arch Gen Psychiatry. 2005;62(6):593-602.

31. Häfner H. Are mental disorders increasing over time? Psychopathology. 1985;18(2-3):66-81.

32. Ngui EM, Khasakhala L, Ndetei D, Roberts LW. Mental disorders, health inequalities and ethics: a global perspective. Int Rev Psychiatry. 2010;22(3):235-244.

33. Regier DA, Narrow WE, Rae DS, Manderscheid RW, Locke BZ, Goodwin FK. The de facto US mental and addictive disorders service system. Epidemiologic catchment area prospective I-year prevalence rates of disorders and services. Arch Gen Psychiatry. 1993;50:85-94.

34. Lin E, Goering P, Offord DR, Campbell D, Boyle MH. The use of mental health services in Ontario: epidemiologic findings. Can J Psychiatry. 1996;41:572-577.

35. Andrews G, Hall W, Teesson M, Henderson S. The Mental Health of Australians. Canberra: Commonwealth Department of Health and Aged Care; 1999.

36. Walsh D, Downe S. Meta-synthesis method for qualitative research: a literature review. J Adv Nurs. 2005;50(2):204-211.

37. Khan N, Bower P, Rogers A. Guided self-help in primary care mental health: meta-synthesis of qualitative studies of patient experience. Br J Psychiatry. 2007;191:206-211.

38. Pound P, Britten N, Morgan M, et al. Resisting medicines: a synthesis of qualitative studies of medicine taking. Soc Sci Med. 2005;61(1): 133-155.

39. Lamb J, Bower P, Rogers A, Dowrick C, Gask L. Access to mental health in primary care: a qualitative meta-synthesis of evidence from the experience of people from 'hard to reach' groups. Health. 2012;16(1): 76-104.

40. Laidlaw A, McLellan J, Ozakinci G. Understanding undergraduate student perceptions of mental health, mental well-being and help-seeking behaviour. Stud Higher Educ. 2015:1-13.

41. Fellmeth G, Plugge E, Paw MK, Charunwatthana P, Nosten F, McGready R. Pregnant migrant and refugee women's perceptions of mental illness on the Thai-Myanmar border: a qualitative study. $B M C$ Pregnancy Childbirth. 2015;15:93. 
42. Shannon PJ, Wieling E, Simmelink-McCleary J, Becher E. Beyond stigma: barriers to discussing mental health in refugee populations. J Loss Trauma. 2015;20(3):281-296.

43. Nemade $\mathrm{N}$ [webpage on the Internet]. Psychology of DepressionBehavioral Theories; 2007. Available from: www.mentalhelp.net. Accessed August 11, 2016.

44. Choudhry FR, Bokharey IZ. Perception of mental health in Pakistani nomads: an interpretative phenomenological analyses. Int J Qual Stud Health Well-Being. 2013;8:22469.

45. Shannon PJ, Wieling E, McCleary JS, Becher E. Exploring the mental health effects of political trauma with newly arrived refugees. Qual Health Res. 2015;25(4):443-457.

46. Kolstad A, Gjesvik N. Collectivism, individualism, and pragmatism in China: implications for perceptions of mental health. Transcult Psychiatry. 2014;51(2):264-285.

47. Bignall WJ, Jacquez F, Vaughn LM. Attributions of mental illness: an ethnically diverse community perspective. Community Ment Health J. 2015;51(5):540-545.

48. Bowers H, Manion I, Papadopoulos D, Gauvreau E. Stigma in schoolbased mental health: perceptions of young people and service providers. Child Adolesc Mental Health. 2013;18(3):165-170.

49. Conner KO, Lee B, Mayers V, et al. Attitudes and beliefs about mental health among African American older adults suffering from depression. J Aging Stud. 2010;24(4):266-277.

50. Nguyen A. Cultural and social attitudes towards mental illness in Ho Chi Minh City, Vietnam. Stanford Univ Res J. 2003;2:27-31.

51. Hailemariam KW. Perceived causes of mental illness and treatment seeking behaviors among people with mental health problems in Gebremenfes Kidus Holy Water Site. Am J Appl Psychol. 2015; 3(2):34-42.

52. Liu CH, Meeuwesen L, van Wesel F, Ingleby D. Why do ethnic Chinese in the Netherlands underutilize mental health care services? Evidence from a qualitative study. Transcult Psychiatry. 2015;52(3): 331-352.

53. Mjøsund NH, Eriksson M, Norheim I, Keyes CL, Espnes GA, Vinje HF. Mental health as perceived by persons with mental disorders-an interpretative phenomenological analysis study. Int J Mental Health Promotion. 2015;17(4):215-233.

54. Diala C, Muntaner C, Walrath C, Nickerson KJ, LaVeist TA, Leaf PJ. Racial differences in attitudes toward professional mental health care and in the use of services. Am J Orthopsychiatry. 2000;70(4): 455-464.

55. Snowden LR. Barriers to effective mental health services for African Americans. Ment Health Serv Res. 2001;3(4):181-187.

56. Miranda J, Cooper LA. Disparities in care for depression among primary care patients. J Gen Intern Med. 2004;19(2):120-126.

57. Al-Darmaki F, Sayed M. Counseling challenges within the cultural context of the United Arab Emirates. In: Gerstein LH, editor. International Handbook of Cross-Cultural Counseling: Cultural Assumptions and Practices Worldwide. Thousand Oaks, CA: Sage; 2009:465-474.

58. Ciftci A, Jones N, Corrigan PW. Mental health stigma in the Muslim community. J Muslim Mental Health. 2013;7(1):17-32.
59. Kopinak J, Berisha B, Mursali B [webpage on the Internet]. An investigation into the health of a representative sample of adults in Kosovo. J Humanit Assistance. 2001. Available from: http://sites.tufts.edu/jha/ files/2011/04/a080.pdf. Accessed November 11, 2015.

60. Suldo SM, Shaffer EJ. Looking beyond psychopathology: the dual-factor model of mental health in youth. School Psych Rev. 2008;37(1):52-68.

61. Association D-AP. Diagnostic and Statistical Manual of Mental Disorders. Arlington: American Psychiatric Publishing; 2013.

62. Lovallo WR. Stress and Health: Biological and Psychological Interactions. Sage Publications; Thousand Oaks; 2015.

63. Siegrist J. Work stress and beyond. Eur J Public Health. 2000;10(3): 233-234.

64. Kivimäki M, Leino-Arjas P, Luukkonen R, Riihimäi H, Vahtera J, Kirjonen J. Work stress and risk of cardiovascular mortality: prospective cohort study of industrial employees. BMJ. 2002;325(7369):857.

65. Zhu C, Chen L, Ou L, Geng Q, Jiang W. Relationships of mental health problems with stress among civil servants in Guangzhou, China. Commun Ment Health J. 2014;50(8):991-996.

66. New York University [webpage on the Internet]. Examine Social Factors to Explain Rise in Diagnoses of Mental Disorders. 2013. Available from: https:/www.sciencedaily.com/releases/2013/04/130425103200. htm. Accessed August 11, 2016.

67. Hansen HB, Donaldson Z, Link BG, et al. Independent review of social and population variation in mental health could improve diagnosis in DSM revisions. Health Aff. 2013;2(5):984-993.

68. Cohen S, Edwards JR. Personality characteristics as moderators of the relationship between stress and disorder. In: Neufeld RW, editor. Advances in the Investigation of Psychological Stress. New York: Wiley; 1989:235-283.

69. Skodol AE. Personality and coping as stress-attenuating or -amplifying factors. In: Dohrenwend BP, editor. Adversity, Stress, and Psychopathology. New York: Oxford University Press; 1998:377-389.

70. Taylor SE, Aspinwall LG. Mediating and moderating processes in psychosocial stress: appraisal, coping, resistance, and vulnerability. In: Kaplan HB, editor. Psychosocial Stress: Perspectives on Structure, Theory, LifeCourse, and Methods. San Diego, CA: Academic Press; 1996:71-110.

71. Zorrilla EP, DeRubeis RJ, Redei E. High self-esteem, hardiness and affective stability are associated with higher basal pituitary-adrenal hormone levels. Psychoneuroendocrinology. 1995;20:591-601.

72. Luthar SS, Cicchetti D, Becker B. The construct of resilience: a critical evaluation and guidelines for future work. Child Dev. 2000;71: 543-562.

73. Leenarts LE, Vermeiren RR, vande VenPM,Lodewijks HP, Doreleijers TA, Lindauer RJ. Relationships between interpersonal trauma, symptoms of posttraumatic stress disorder, and other mental health problems in girls in compulsory residential care. J Trauma Stress. 2013;26(4):526-529.

74. Patel V. Explanatory models of mental illness in sub-Saharan Africa. Soc Sci Med. 1995;40(9):1291-1298.

75. Cohen S, Underwood LG, Gottlieb BH. Social Support Measurement and Intervention: A Guide for Health and Social scientists. New York, NY: Oxford University Press; 2000
Neuropsychiatric Disease and Treatment

\section{Publish your work in this journal}

Neuropsychiatric Disease and Treatment is an international, peerreviewed journal of clinical therapeutics and pharmacology focusing on concise rapid reporting of clinical or pre-clinical studies on a range of neuropsychiatric and neurological disorders. This journal is indexed on PubMed Central, the 'PsycINFO' database and CAS,
Dovepress

and is the official journal of The International Neuropsychiatric Association (INA). The manuscript management system is completely online and includes a very quick and fair peer-review system, which is all easy to use. Visit http://www.dovepress.com/testimonials.php to read real quotes from published authors. 